\title{
METASTABLE CONFIGURATIONS OF WIGNER CRYSTALS IN A CIRCULAR TRAP
}

\author{
E. Anisimovas, O. Rancova, and T. Varanavičius \\ Vilnius University, Saulètekio 9, LT-10222 Vilnius, Lithuania \\ E-mail: egidijus.anisimovas@ff.vu.lt
}

Received 14 September 2010; revised 27 October 2010; accepted 15 December 2010

\begin{abstract}
We model the formation of ordered structures in systems consisting of up to 52 identical particles interacting by Coulomb repulsion forces and confined within a two-dimensional parabolic trap. Our algorithm consists of a number of Metropolis steps followed by the steepest-descent minimization of the total potential energy of the system. The role of the first (Metropolis) stage is to create a random canonically distributed configuration, while the subsequent minimization locates the closest local minimum starting from this random configuration. In most cases we find that more than one stable configuration may be formed, and often the lowest-energy configuration is not the most probable one. The concept of configurational entropy is introduced to quantify the uncertainty due to the availability of several alternative structures.
\end{abstract}

Keywords: Wigner crystallization, Monte Carlo simulation, entropy

PACS: 05.20.-y, 61.46.Bc

\section{Introduction}

The formation of ordered structures in systems consisting of a finite number of strongly interacting particles is of considerable interest for researchers working in diverse areas of physics, in particular, low dimensional nanostructures [ $[1-3]$ and plasma physics [4-6]. In line with tradition, such structures are often referred to as Wigner crystals as it was Eugene Wigner who proposed, back in 1934, that a crystal lattice may be formed by an electron gas of sufficiently low density [7].

As a matter of fact, the formation of a threedimensional lattice of electrons was never realized, however, its two-dimensional analogue turns out to be feasible in electron systems confined on the surface of liquid helium $[1,8]$ as well as in semiconductor heterostructures [9, 10].

In recent years, investigations of ordered structures have been gradually shifting from electronic systems towards systems consisting of larger and heavier particles. In particular, most of recent successful experiments have been performed with micrometre-sized particles carrying electrostatic charges of around several thousand elementary units that are formed in a radio-frequency discharge and captured by electrostatic traps $[5,6]$.

The underlying physics of these systems is the same as envisioned by Wigner as the crystallization is still induced by the domination of the Coulomb repulsion energy over the kinetic energy. On the other hand, the time and length scales are much more advantageous for direct observation [6]. The trajectories of microparticles can be recorded with the aid of ordinary CCD cameras, and typical periods of their normal-mode oscillations are on the order of seconds [11]. The behaviour of these crystals can be analysed within a completely classical or quasiclassical approach [12].

The most basic issue pertaining to the study of static properties of Wigner crystals is the determination of energetically stable configurations formed by a certain number of particles captured in a trap of a given shape and dimensionality.

It turns out that in typical situations one finds not one but several distinct stationary configurations, and the number of such configurations grows very rapidly (exponentially) with the number of particles [4]. The stationary configuration with the lowest energy corresponds to the global energy minimum and is commonly referred to as the ground state. Other configurations corresponding to local energy minima and characterized by higher energy values are known as metastable states.

In order to give an illustration to what has just been said, in Fig. 1 we show the two competing configu- 


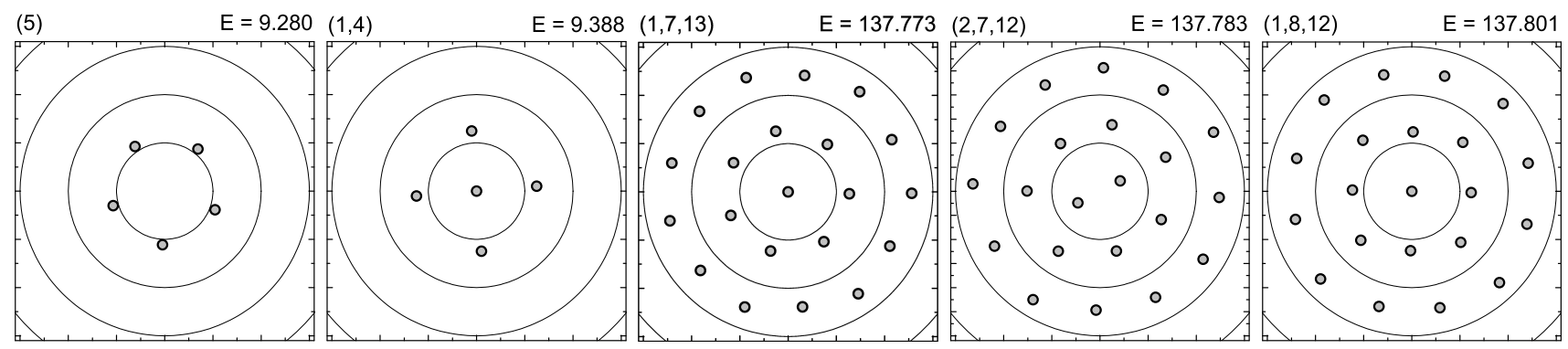

Fig. 1. Ground and metastable configurations of five- and 21-particle systems. The concentric circles mark equipotential lines of the parabolic trap.

rations present in a five-particle system and the three competing configurations that we have found in a system of 21 particle. The configurations are arranged in the order of increasing energy and are marked by their shell-structure. One can clearly see in the graphs that due to the circular shape of the confining potential particles tend to arrange themselves into nearly circular shells. Thus, is it handy to identify configurations by enumerating the numbers of particles in each shell starting with the innermost one. For example, the ground state configuration of 21-particle system is $(1,7,13)$, while the arrangement of particles in two metastable states is $(2,7,12)$ and $(2,8,11)$. We note, however, that when the number of particles exceeds 30 it is not always possible to discern the shell structure in some configurations. The reason for that is the competition between the circular confinement and the hexagonal lattice symmetry that appears in the interior of the trap.

Simulation studies often concentrate on the quest to determine the global potential energy minimum. Various algorithms, based on the simulated annealing [13] and dissipative molecular dynamics [4, 14] are constructed and applied. A certain uneasiness is felt when the optimal configuration obtained in a simulation does not reproduce that observed experimentally.

However, recent experiments performed on threedimensional particle clusters [ [ ] have shown that metastable states may be formed with significantly higher probabilities than the ground state. It has been argued that this happens when a metastable state controls a larger basin of attraction. Here, the meaning of the word "basin" is best portrayed as the drainage basin of a river, that is, the area from which a river collects its waters.

In order to illustrate this concept we present Fig. 2. This figure shows a simple one-dimensional sketch of a certain function intended to represent the potential energy. This function has a global minimum (marked by GM on the graph) which is situated at the bottom of a rather narrow valley and is thus characterized by a small basin of attraction. In contrast, the local mini-

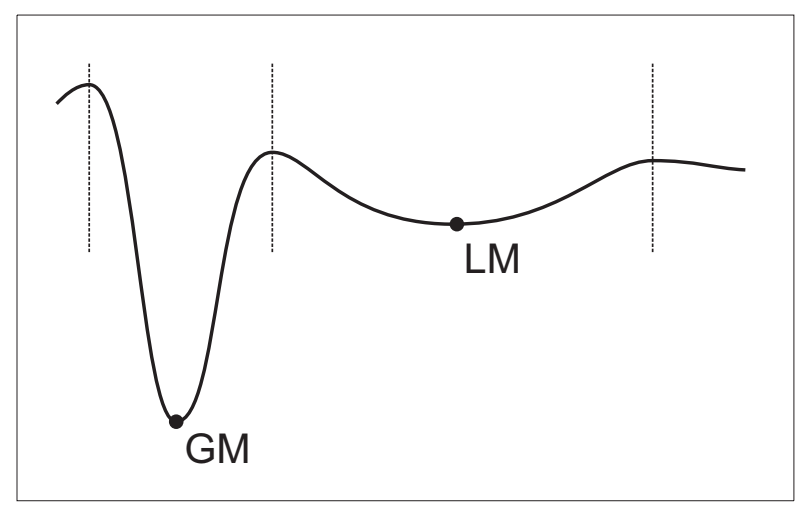

Fig. 2. One-dimensional function with two minima. The global minimum (GM) controls a smaller basin of attraction than the local minimum (LM). Vertical dotted lines delimit the different basins.

mum (LM) is much more shallow, and its basin of attraction is much larger. The basins of attraction are delimited by vertical lines. It is clear that a particle placed at a random point and allowed to roll down towards the nearest stationary point would most likely end up in a metastable state.

The phase space defined by all possible configurations of $N$ two-dimensional particles is $2 N$-dimensional and, undoubtedly, the distribution of its minima and separating barriers is rather complicated. However, it is still possible to analyse the phase space by locating the energy minima and partitioning the phase space into a set of basins of attraction adjacent to these minima. The definition of the basin of attraction in many dimensions is as simple as in one dimension: a given point $\mathcal{P}$ belongs to the basin of attraction of a stable configuration $\mathcal{C}$ if a straightforward downhill minimization algorithm starting from the point $\mathcal{P}$ converges to the minimum $\mathcal{C}$.

The goal of the present work is to perform a numerical study in order to determine all (both ground and metastable) stationary states in a system of up to 52 charged particles in a two-dimensional parabolic trap, and thus gain insight into the occurrence probabilities of various minima. We propose a quantitative measure for the probabilities, and introduce a useful concept of configurational entropy. 


\section{Model and computational approach}

Let us consider a system consisting of a given number $N \leqslant 52$ of identical two-dimensional particles of mass $m$ and electrostatic charge $q$. The in-plane motion of particles is confined by the trap potential which shall be assumed to be isotropic and of a parabolic shape with the characteristic confining frequency denoted by $\omega$. Thus the classical Hamilton function of the system may be written as

$$
\mathcal{H}=\sum_{i=1}^{N} \frac{\boldsymbol{p}_{i}^{2}}{2 m}+\sum_{i=1}^{N} \frac{m \omega^{2} \boldsymbol{r}_{i}^{2}}{2}+\sum_{i>j}^{N} \frac{q^{2}}{4 \pi \epsilon_{0}\left|\boldsymbol{r}_{i}-\boldsymbol{r}_{j}\right|} .
$$

Here, the two-dimensional vectors $\boldsymbol{r}_{i}$ and $\boldsymbol{p}_{i}$ stand, respectively, for the radius-vector and the momentum of the $i$ th particle. The last term of the Hamilton function (1) represents the pairwise Coulomb interactions between the particles.

Looking for stationary equilibrium configurations, the kinetic energy term is discarded and one is concerned with the minimization of the total potential energy which is given by the second and the third term of Eq. (1). Obviously, these two terms are in competition: the Coulomb repulsion tends to spread the particles as far apart as possible while the confinement tries to hold them together.

Convenient scaling properties of the potential are easy to spot and exploit. It may be shown that by using proper units for the coordinates and the energies the problem is reformulated as the minimization of the dimensionless interaction energy

$$
E=\frac{1}{2} \sum_{i=1}^{N} \boldsymbol{r}_{i}^{2}+\sum_{i>j}^{N} \frac{1}{\left|\boldsymbol{r}_{i}-\boldsymbol{r}_{j}\right|},
$$

which will be our main concern hereafter.

It is interesting to observe that our model closely resembles the so-called Thomson problem which considers the equilibrium distribution of a number $N$ of equal charges on a sphere. This problem stems from the Thomson model of the atom proposed in 1904 and is of more academic nature. Nevertheless, the Thomson problem recently attracted considerable attention. Ground state configurations have been studied [15, 16] mostly concentrating on their energies and symmetry properties.

\subsection{Algorithm}

The algorithm employed to determine the stable states and estimate their occurrence probabilities (as a measure of the adjacent basin of attraction) is a combination of a sequence of standard Metropolis steps [ 17 , 18] followed by the steepest-descent [19] energy minimization. A similar approach was used before in a three-dimensional set-up [4].

Let us briefly remind the reader that a Metropolis step is a possibility for the simulated system to perform a change of its current configuration. A small random modification of the coordinates of one of the particles is proposed (by a Mersenne twister [20] random number generator) and either accepted or rejected. The probability of acceptance may be written as

$$
p=\min \left(1, \mathrm{e}^{-\Delta E / k T}\right) .
$$

Here, $\Delta E$ is the change in the total potential energy of the system due to the proposed move, and the function $\min (a, b)$ selects the smaller value of its two arguments. In simple terms this means that the changes that lower the energy are accepted always, while the steps upwards in the energy scale are accepted with an exponentially decaying probability.

The quantity $k T$ is the "temperature" of the simulation. When this temperature is high the simulated system is more likely to perform moves that increase its energy. Note, that normally temperatures and energies are measured in different units and the Boltzmann constant $k$ is used as a factor of unit conversion. In our case, since we work in dimensionless units we treat $k T$ as an indivisible symbol and simply call it the temperature.

When a sufficiently large number of Metropolis steps have been performed (we typically use $10^{3}$ to $10^{4}$ steps in our work) the canonical distribution is established. Then the current configuration of the system is, in essence, randomly drawn from all possible configurations with the probability proportional to the Boltzmann factor $\mathrm{e}^{-E / k T}$.

The next stage of the algorithm is to make the system to roll down to the nearest energy minimum, either global or local. This is achieved by employing the steepest descent minimum search supplemented by the parabolic extrapolation [22].

The above cycle of two stages - thermalization and sudden cooling - is repeated a number (typically $10^{4}$ to $10^{5}$ ) of times and statistics of the minima found is collected. In this way we are able to calculate the probabilities that the configuration of our system will be within the basin of attraction of a given minimum. Also, having repeated the described numerical experiment a large number of times at different temperatures we are confident that the complicated multidimensional phase space of configurations is explored reasonably well and 


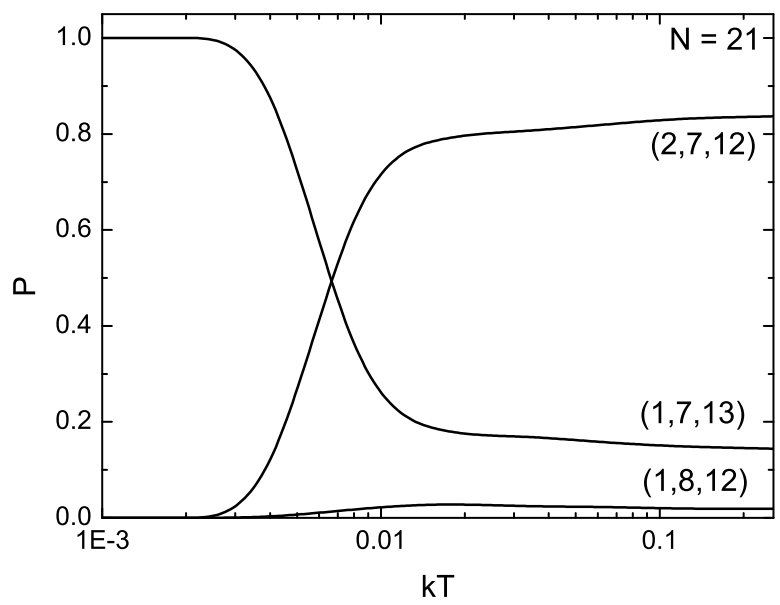

Fig. 3. Typical temperature dependences of the probabilities. The case of 21 particles in a trap is shown. Note that the ground state

$(1,7,13)$ is not the most probable at high temperatures.

all stable states that have realization probabilities higher than $10^{-5}$ have been determined.

Let us study a typical result. Figure $\beta$ shows the case of $N=21$ electrons in a trap. As we mentioned before, three stable configurations are possible and can be labeled by their shell structure.

When the temperature of simulation is low, the system can oscillate randomly only in the vicinity of the global minimum. Thus, Fig. 3 shows that as long as $k T \lesssim 3 \cdot 10^{-3}$ the global-minimum configuration $(1,7,13)$ is found with probability close to unity, and the remaining two stable configurations remain unreachable.

As the temperature of the simulation increases it becomes possible to explore larger areas of the configurational phase space and the system may cross the potential barriers into the basins of attraction of the other minima. Evidently, the metastable state $(2,7,12)$ is at the bottom of a substantially larger basin of attraction, therefore, its probability becomes the largest.

Note that at high temperatures the temperature dependences of the probabilities pertaining to various minima level off. That means that the temperature has become significantly higher than the differences between the minima and separating barrier heights. Thus, the high-temperature limit of the studied probability is a well defined and temperature-independent measure of the phase-space volume surrounding different minima.

\section{Results}

With this preparatory material in our background let us turn to the obtained results.

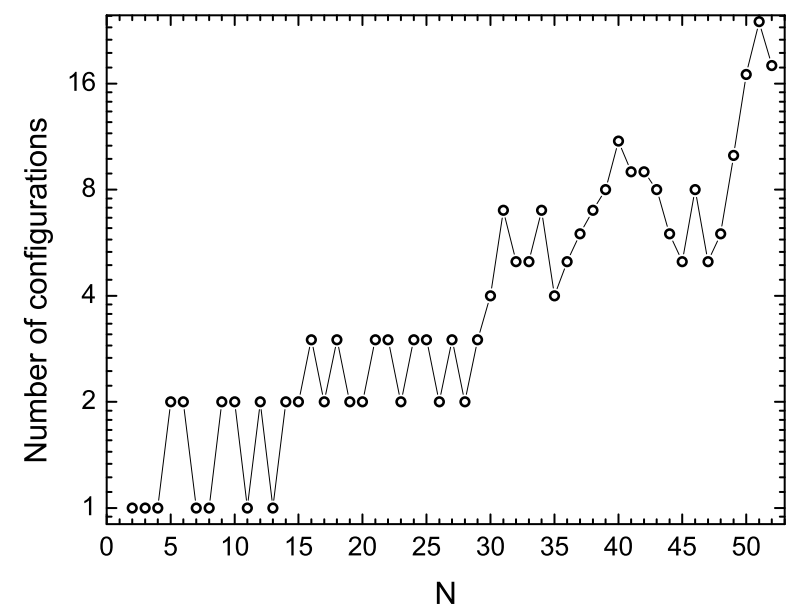

Fig. 4. Number of stable configurations of a $N$-particle system in a trap.

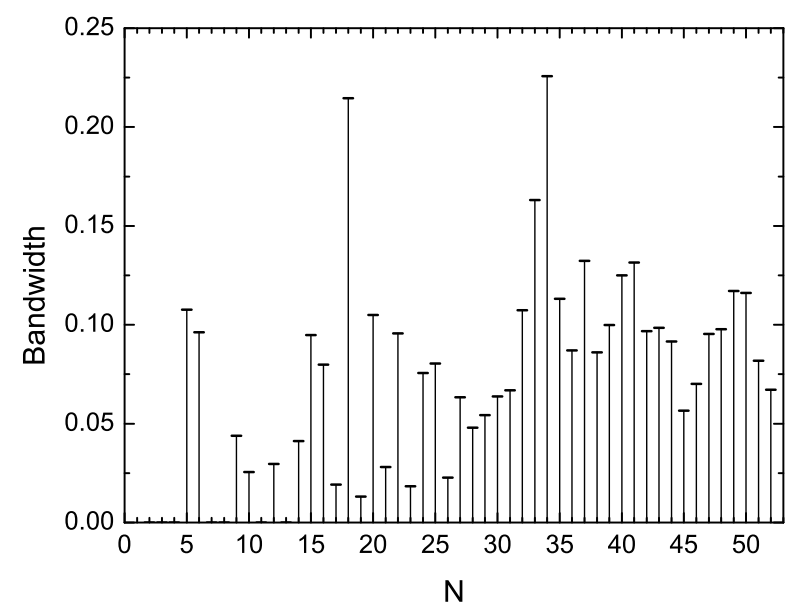

Fig. 5. Energy bandwidth in a $N$-particle system.

Figure 4 shows the dependence of the number of stable configurations on the number of particles in a circular two-dimensional trap. We see that only systems consisting of $N=2,3,4,7,8,11$, and 13 particles have uniquely defined configurations. As long as the number of particles does not exceed $N=29$ one has up to three stable configurations, and afterwards the number of configurations takes off. One sees that in general the number of configurations grows rather rapidly, and this growth is quite erratic.

On the other hand, in Fig. 5 we show the width of the energy band. This width is defined as the difference of the highest and the lowest possible energies among all stable configurations. In other words, it is the range of available energies. We see that this dependence is also rather erratic, however, there is no visible widening of the band. Thus, as the number of particles in the trap increases, various stationary states become closer and closer in energy and eventually become impossible to 

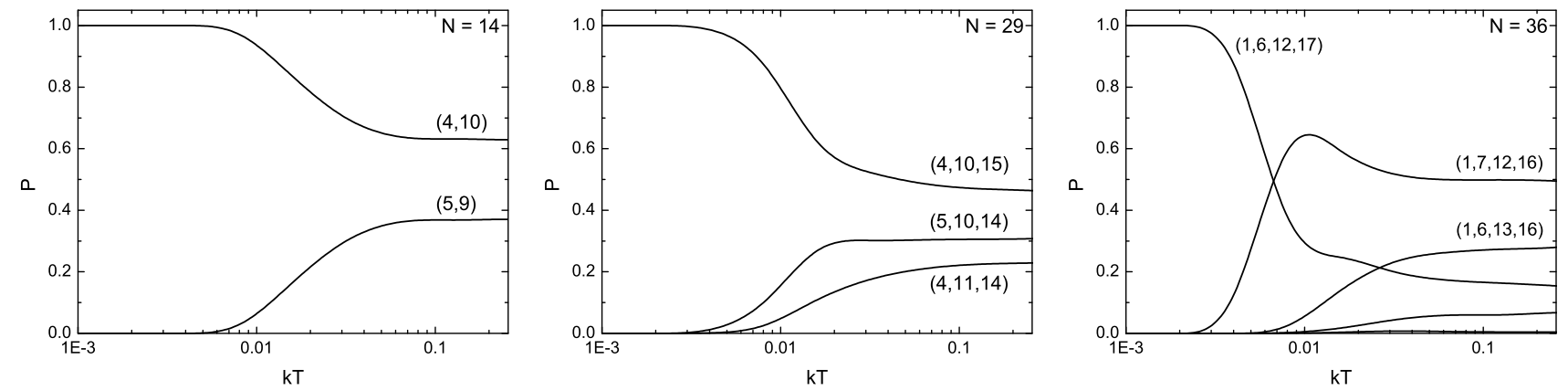

Fig. 6. Typical temperature dependences of the probability.

separate. As a consequence, for larger systems, when the number of distinct stable states reaches several tens or more, it makes little sense to study individual configurations and the useful concept that survives is the energy band.

Another very important point to note is that the realization probabilities of metastable states may be very different. Let us take a look at Fig. 6 where we show three typical behavioural patterns of the temperature dependence of probabilities to end up in a given stable state. The three panels correspond to traps containing 14, 29, and 36 particles. Figure 3 that we considered earlier as an illustration of the algorithm may also be regarded as a supplement of this figure.

A 14-particle system has only two stable configurations, which in terms of their shell structure are labeled $(4,10)$ for the ground state and $(5,9)$ for the metastable state. Naturally, at low temperatures $k T \lesssim 0.01$ only the ground state is available while at higher temperatures both states may be realized with comparable probabilities.

A system of 29 particles has three stable configurations which are, in the order of increasing energy: $(4,10,15),(5,10,14)$, and $(4,11,14)$. As one can see from the corresponding graph, in the high-temperature limit the probabilities of all three states are of comparable magnitude. The ground state has the highest probability, followed by the first excited state, and then followed by the second excited state that has the highest energy.

However, such a clear-cut arrangement is not always realized. The last panel pertaining to the 36-particle system illustrates the point. Here one finds in total five stable configurations. At high temperatures the most probable is not the ground state $(1,6,12,17)$ but the second one $(1,7,12,16)$. The third lowest state is $(1,6,13,16)$ and at high temperatures also becomes more probable than the ground state. The remaining two configurations - $(1,7,13,15)$ and $(1,7,11,17)$ - have rather low probabilities. The probability of the highest-energy configuration does not exceed 0.005 .

In general, as the number of particles in the trap and the number of stable configurations grow, only a few most important states are dominant while the basins of attraction of the remaining ones account for a very small fraction of the phase-space volume. This observation permits one to conclude that the number of states is not such a useful concept. Simple counting of the number of found stationary configurations is misleading in two ways. First of all, since the number of performed simulation runs is always finite, some of the less probable configurations may be overlooked or discovered by accident. Thus, the total number of configurations is usually not known with confidence. Moreover, straightforward counting of configurations disregards their wildly different probabilities and importance.

Therefore, we propose to rely on the concept of configurational entropy. Conforming to the definitions accepted in information theory [21] we define the configurational entropy as

$$
S=-\sum_{j} p_{j} \log _{2} p_{j} .
$$

Here, the sum is taken over all states enumerated by the index $j$ and the symbols $p_{j}$ denote the probabilities of these states. We find it convenient to take the logarithm to the base 2 in Eq. (4).

The proposed configurational entropy is the measure of uncertainty of the particle configurations. We note, that if there is only one available configuration, the entropy equals zero. This reflects the fact that we know the configuration that will be found in any experiment and consequently there in no uncertainty. Further, the entropy (4) is stable with respect to an unexpected discovery of an additional state of low probability.

Temperature dependences of the configurational entropy are shown in Fig. 7 (a) for several values of the number of particles in the trap. Since the entropy is a 

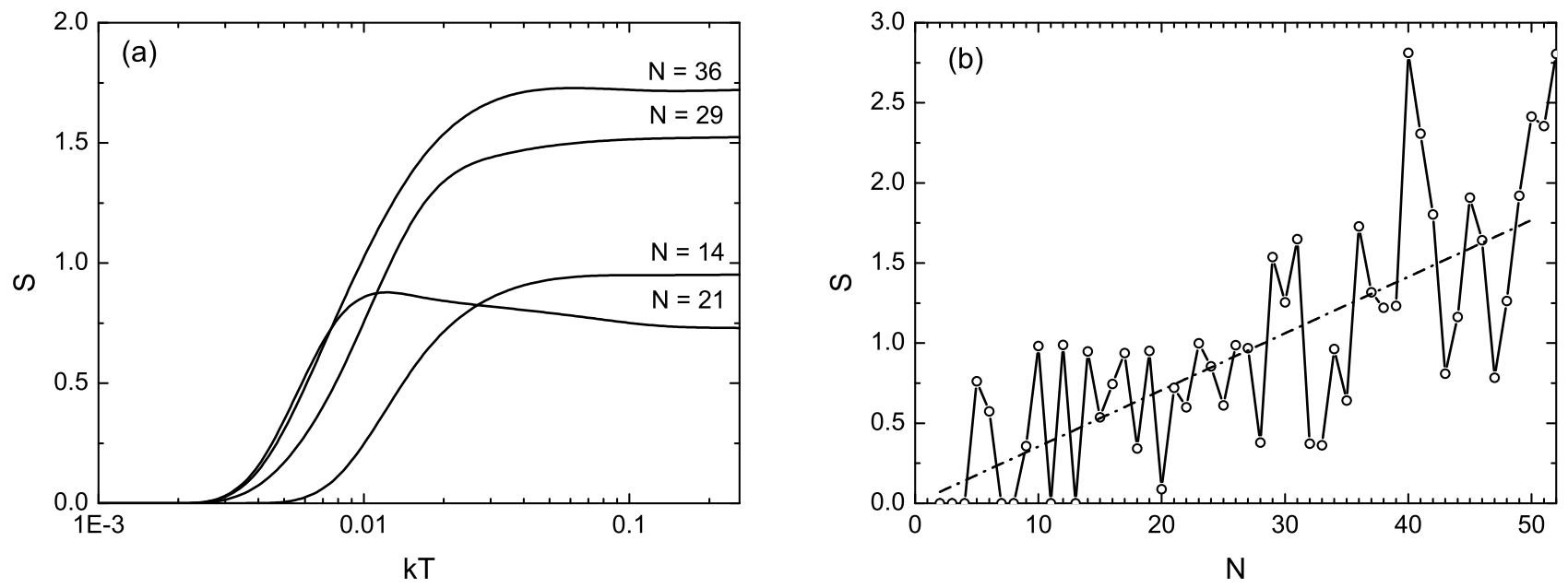

Fig. 7. Configurational entropy of a Wigner crystal in a circular trap. Panel (a) shows the temperature dependence of the configurational entropy for several values of the number of particles. Panel (b) displays the dependence of the high-temperature limit of the entropy on the number of particles.

function of the probabilities, these dependences repeat the general shape of the probability dependences. At low temperatures the entropy equals zero, and the dependences level off at high temperatures when the probabilities of all configurations become temperature independent. Thus, the high-temperature limit of the entropy is a useful measure of the configurational uncertainty. The right panel, Fig. 7(b), displays the dependence of the configurational entropy on the number of particles in a trap. The growth of the entropy is erratic. The dotted line shows the optimal linear fit, however, we see that the available data is insufficient to tell for sure whether this growth is linear on the average or not. We find this question interesting and hope to further investigate it in our forthcoming work.

\section{Acknowledgements}

Olga Rancova is a postdoctoral fellow funded by the Lithuanian Research Council. Tadas Varanavičius acknowledges the Summer Undergraduate Research Fellowship Award from the Lithuanian Research Council.

\section{References}

[1] E. Rousseau, D. Ponarin, L. Hristakos, O. Avenel, E. Varoquaux, and Y. Mukharsky, Addition spectra of Wigner islands of electrons on superfluid helium, Phys. Rev. B 79, 045406 (2009).

[2] F. Cavaliere, U. De Giovannini, M. Sassetti, and B. Kramer, Transport properties of quantum dots in the Wigner molecule regime, New J. Phys. 11, 123004 (2009)
[3] R. Côté, J.-F. Jobidon, and H.A. Fertig, Skyrme and Wigner crystals in graphene, Phys. Rev. B 78, 085309 (2008).

[4] H. Kählert, P. Ludwig, H. Baumgartner, M. Bonitz, S. Käding, D. Block, A. Melzer, and A. Piel, Probability of metastable configurations in spherical threedimensional Yukawa crystals, Phys. Rev. E 78, 036408 (2008).

[5] T.E. Sheridan and K.D. Wells, Dimensional phase transition in small Yukawa clusters, Phys. Rev. E 81, 016404 (2010).

[6] M. Bonitz, C. Henning, and D. Block, Complex plasmas: a laboratory for strong correlations, Rep. Prog. Phys. 73, 066501 (2010).

[7] E. Wigner, On the interaction of electrons in metals, Phys. Rev. 46, 1002 (1934).

[8] C.C. Grimes and G. Adams, Evidence for a liquid-tocrystal phase transition in a classical, two-dimensional sheet of electrons, Phys. Rev. Lett. 42, 795 (1979).

[9] E.Y. Andrei, G. Deville, D.C. Glattli, and F.I.B. Williams, Observation of a magnetically induced Wigner solid, Phys. Rev. Lett. 60, 2765 (1988).

[10] Y.P. Chen, G. Sambandamurthy, Z.H. Wang, R.M. Lewis, L.W. Engel, D.C. Tsui, P.D. Ye, L.N. Pfeiffer, and K.W. West, Melting of a 2D quantum electron solid in high magnetic field, Nature Phys. 2, 452 (2006).

[11] Y. Ivanov and A. Melzer, Modes of three dimensional dust crystals in dusty plasmas, Phys. Rev. E 79, 036402 (2009).

[12] E. Anisimovas, A. Matulis, M.B. Tavernier, and F.M. Peeters, Power-law dependence of the angular momentum transition fields in few-electron quantum dots, Phys. Rev. B 69, 075305 (2004).

[13] W. Yang, K. Nelissen, M. Kong, Z. Zeng, and F.M. Peeters, Structure of binary colloidal systems con- 
fined in a quasi-one-dimensional channel, Phys. Rev. E 79, 041406 (2009).

[14] S.W.S. Apolinario and F.M. Peeters, Melting of anisotropically confined Coulomb balls, Phys. Rev. B 78, 024202 (2008).

[15] E.L. Altschuler and A. Pérez-Garrido, Defect-free global minima in Thomson's problem of charges on a sphere, Phys. Rev. E 73, 036108 (2006).

[16] J.R. Morris, D.M. Deaven, and K.M. Ho, Geneticalgorithm energy minimization for point charges on a sphere, Phys. Rev. B 53, 1740 (1996).

[17] N. Metropolis, A.W. Rosenbluth, M.N. Rosenbluth, A.H. Teller, and E. Teller, Equation of state calculations by fast computing machines, J. Chem. Phys. 21, 1087 (1953).

[18] D.P. Landau and K. Binder, A Guide to Monte Carlo
Simulations in Statistical Physics, 3rd ed. (Cambridge University Press, 2009).

[19] W.H. Press, S.A. Teukolsky, W.T. Vetterling, and B.P. Flannery, Numerical Recipes: The Art of Scientific Computing, 3rd ed. (Cambridge University Press, 2007).

[20] M. Matsumoto and T. Nishimura, Mersenne Twister: A 623-dimensionally equidistributed uniform pseudorandom number generator, ACM Trans. Model. Comput. Simulat. 8, 3 (1998).

[21] D.J.C. MacKay, Information Theory, Inference and Learning Algorithms (Cambridge University Press, 2003).

[22] A. Matulis, D. Jarema, and E. Anisimovas, A quasiclassical approach to strongly correlated quantum dots, Cent. Eur. J. Phys. 7, 704 (2009).

\title{
METASTABILIOS VIGNERIO KRISTALŲ KONFIGŪRACIJOS APSKRITOJE GAUDYKLE்JE
}

\author{
E. Anisimovas, O. Rancova, T. Varanavičius \\ Vilniaus universitetas, Vilnius, Lietuva
}

\section{Santrauka}

Tiriamas tvarkingų darinių formavimasis sistemose, sudarytose iš $N \leqslant 52$ identiškų dalelių, esančiu apskritoje dvimatèje gaudyklèje ir tarpusavyje sąveikaujančiu Kulono stūmos jègomis. Skaitmeniniam modeliavimui pasitelkiami Metropolio algoritmas ir greičiausio nusileidimo metodas. Metropolio algoritmo paskirtis yra sugeneruoti atsitiktinę (kanoninio pasiskirstymo) daleliu padèčiu konfigūraciją. Tuo tarpu greičiausio nusileidimo metodas leidžia aptikti artimiausią sąveikos energijos minimumą atitinkančią stabilią konfigūraciją. Daugeliu atvejų tokių konfigūracijų randame keletą ir žemiausios energijos konfigūracija (pagrindinè sistemos būsena) dažnai nèra labiausiai tikètina. Siekiant kiekybiškai ivertinti sistemos struktūros neapibrež̌tumą, atsirandantị dẻl keleto konkuruojančių konfigūracijų buvimo, îvedama konfigūracinès entropijos sąvoka. 